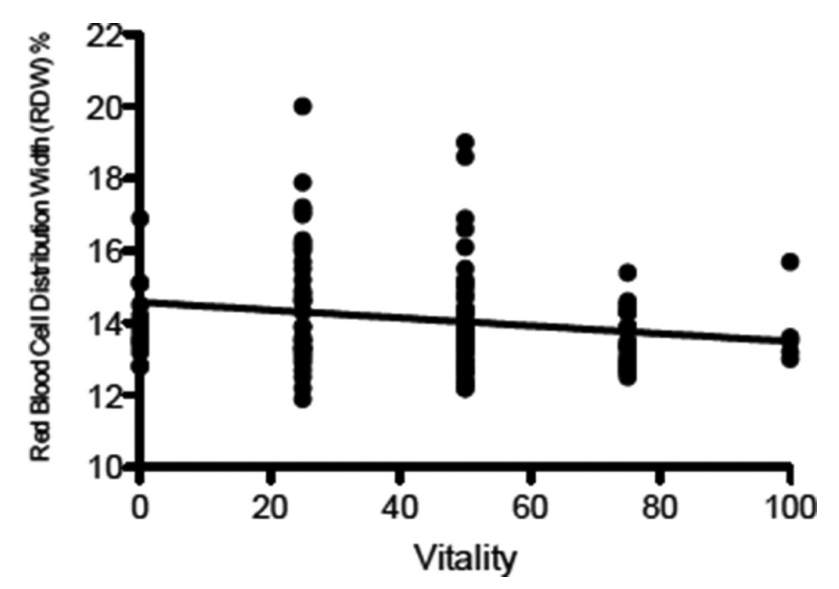

Abstract S7D:7 Figure 2 Vitality score (measured in LUPUS Qol) and RDW in adults with SLE (UCLH cohort)

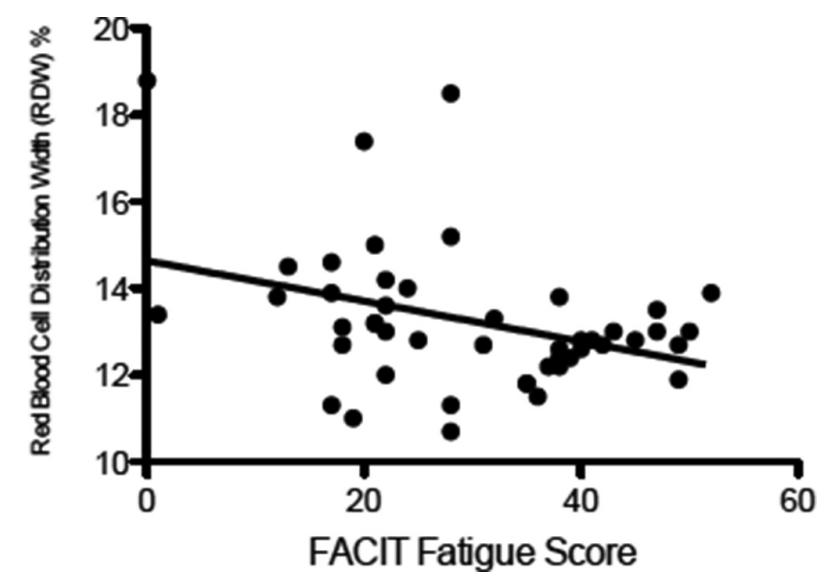

Abstract S7D:7 Figure 3 FACIT fatigue score and RDW in adults with SLE (Australian cohort)

This includes a score of vitality level (a lower score is suggestive of more fatigue). RDW was recorded, in addition to standard markers of lupus disease activity including Erythrocyte Sedimentation Rate (ESR), Complement C3, anti-double-stranded DNA binding (anti-dsDNA), C-reactive protein (CRP) and SLEDAI/ BILAG. Spearman's rank was used to analyse variables with a $\mathrm{p}$ value of $<0.05$ considered significant.

Results In cohort 1, 72 patients aged 14-42 (median 21) were recruited. FACIT score did not correlate with anti-dsDNA $(p=0.4)$, C3 $(p=0.06)$, ESR $(p=0.06)$, CRP $(p=0.1)$ or SLEDAI $(p=0.6)$. There was a strongly significant correlation between FACIT and RDW ( $\leq \leq 0.001 ; \mathrm{r}=-0.44)$; figure 1 . In cohort 2, 106 patients were recruited aged 18-75 (median 44.5). RDW correlated with ESR ( $\mathrm{p}=0.03 ; \mathrm{r}=-0.20)$, BILAG $(\mathrm{p}=0.002 ; \mathrm{r}=-0.30)$ and vitality scores $(\mathrm{p}=0.02 ; \mathrm{r}=0.23)$; figure 2. In cohort 3, 47 patients aged 19-75 (median 46) were recruited. FACIT correlated with RDW $(\mathrm{p}=0.03 ; \mathrm{r}=-0.32)$; figure 3.

Conclusions An elevated RDW correlates with higher levels of fatigue. For the first time a serologically marker has shown strong association with fatigue in patients with lupus. This is demonstrated in three groups of varying age, ethnicity and geography and using two different fatigue scores.
Poster session 1: Autoantibodies, biomarkers and imaging (1)

\section{PS1:1 HIGH TYPE I INTERFERON ACTIVITY IS ASSOCIATED WITH ACTIVE CLASS 3/4 LUPUS NEPHRITIS IN EUROPEAN-AMERICAN LUPUS PATIENTS INDEPENDENT OF ANTI-DSDNA ANTIBODIES}

${ }^{1} \mathrm{~T}$ Iwamoto, ${ }^{2} \mathrm{JM}$ Dorschner, ${ }^{1} \mathrm{MA}$ Jensen, ${ }^{2} \mathrm{D}$ Vsetecka, ${ }^{2} \mathrm{~S}$ Amin, ${ }^{2} \mathrm{~A}$ Makol, ${ }^{2} \mathrm{FC}$ Ernste, ${ }^{2} \mathrm{~T}$ Osborn, ${ }^{2} \mathrm{~K}$ Moder, ${ }^{2} \mathrm{~V}$ Chowdhary, ${ }^{1} \mathrm{~TB}$ Niewold. ${ }^{1}$ Colton Centre for Autoimmunity, New York University, New York, USA; ${ }^{2}$ Mayo Clinic College of Medicine, Rochester, USA

10.1136/lupus-2018-abstract.50

Background/purpose Lupus nephritis (LN) is one of the most severe types of organ involvement in systemic lupus erythematosus (SLE), despite the recent advances in immunosuppressive therapies. High type 1 interferon (IFN) is a heritable risk for SLE, and some previous studies have suggested a link between high IFN and lupus nephritis. However, little is known about the relationships between high levels of IFN and the subtypes of LN, and whether IFN is more associated with anti-dsDNA antibodies or with clinical nephritis.

Methods We studied 244 European-American (EA) SLE patients and measured type 1 IFN in sera by performing WISH IFN bioassay as described previously. Subtypes of LN were confirmed by renal biopsy review. Complements, antidsDNA and other auto-antibodies were measured in the clinical laboratory, and standard clinical cut-offs were used to define a positive result. Non-parametric analyses were used to compare IFN data with the clinical data.

Results IFN level and SLEDAI score was positively correlated $(\mathrm{r}=0.26, \mathrm{p}<0.0001$, Spearman $)$ in our cross-sectional evaluation. EA subjects with a high levels of IFN (IFN score >2) were more likely to have renal manifestations compared to the subjects with a low levels of IFN (IFN score <2) $(\mathrm{p}<0.001, \mathrm{OR}=3.0$, Fisher's exact test). In addition, the incidence rate of class $3 / 4 \mathrm{LN}$ was significantly higher among patients with a high levels of IFN compared to the patients with low levels of IFN ( $\mathrm{p}<0.01, \mathrm{OR}=5.5$, Fisher's exact test). Notably, IFN level was significantly higher in active class $3 / 4$ LN compared to inactive class $3 / 4 \mathrm{LN}(\mathrm{p}<0.05$ Mann-Whitney $\mathrm{U})$ and this was not observed in non-class $3 / 4 \mathrm{LN}$ populations. Positivity of ds-DNA antibody did not show significant difference between inactive class $3 / 4 \mathrm{LN}$ and active class $3 / 4 \mathrm{LN}$. Conclusion Our data support an association between type 1 IFN and class 3/4 nephritis that is independent of overall SLEDAI and anti-dsDNA antibodies, suggesting that IFN is involved in renal pathogenesis. These data also suggest that IFN could predict renal disease activity or the future risk of developing LN, especially class $3 / 4 \mathrm{LN}$ in EA SLE patients.

\section{PS1:2 DEVELOPMENT OF A MULTIMARKER MODEL FOR THE DETECTION OF SYSTEMIC LUPUS ERYTHEMATOSUS BASED ON NEW AND TRADITIONAL AUTOANTIBODIES}

${ }^{1} \mathrm{P}$ Budde, ${ }^{1} \mathrm{H}-\mathrm{D}$ Zucht, ${ }^{2} \mathrm{~T}$ Witte, ${ }^{3} \mathrm{M}$ Schneider, ${ }^{1} \mathrm{P}$ Schulz-Knappe. ${ }^{1}$ Protagen $A G$, Dortmund, Germany, Dortmund, Germany; ${ }^{2}$ Clinical Immunology and Rheumatology, Hannover, Germany; ${ }^{3}$ Policlinic for Rheumatology and Hiller Research Centre for Rheumatology, Heinrich-Heine-University Dusseldorf, Germany

10.1136/lupus-2018-abstract.51 